\title{
Long-term observations of the classical nova X Ser and the activity of post-novae
}

\author{
Vojtěch Šimon* \\ Astronomical Institute, The Czech Academy of Sciences, 25165 Ondřejov, Czech Republic \\ Czech Technical University in Prague, Faculty of Electrical Engineering, Prague, Czech \\ Republic \\ E-mail: simoneasu.cas.cz
}

\begin{abstract}
We show a relation of the classical nova outbursts and the activity of post-novae. A variety of the optical activities of post-novae exists. Also large changes of the type of activity in the decades after the classical nova outburst are observed in some of them. Although the ensemble of systems in this analysis is incomplete, the similarities in the decaying branches of the outbursts in some post-novae are the features favoring the dwarf nova outbursts. The features consistent with the dwarf nova outbursts appear mainly in the later phases (years, decades) of the gradual decay of the mean luminosity of some post-novae. This suggests that they contain the accretion disks which were able to switch from the hot (ionized) state to the thermal-viscous instability (TVI) regime. Such a TVI of the disk can appear in some post-novae even during the first century after the classical nova outburst (often sooner after the classical nova outburst in cataclysmic variables with longer orbital periods). Activity of the mass-donating star in the brightness variations of some post-novae can be caused by the appearance and changes of the position of the active regions on the donor.
\end{abstract}

The Golden Age of Cataclysmic Variables and Related Objects V (GOLDEN2019)

2-7 September 2019

Palermo, Italy

*Speaker. 


\section{Introduction}

Explosion of a classical nova is caused by episodic hydrogen burning of the accreted matter on the white dwarf (WD) in a cataclysmic variable (CV) [46]. The source of nova can be a CV with the accretion disk or a polar. Polars are CVs with strongly magnetized WD ( $B>10^{7}$ Gauss), without the accretion disk. Cyclotron, thermal and bremsstrahlung emissions come from accretion column onto the WD (e.g. [46]). The magnetic field of the WD strongly influences the activity of $\mathrm{CV}$, so it will play a role in the activity of a post-nova.

\section{X Ser}

X Ser erupted as a classical nova in 1903 [23, 7]. It was classified by [7] as a nova of type D. Its orbital period $P_{\text {orb }}$ is quite long, $1.478 \mathrm{~d}$ [43].

We used over 100 years of photometry (the data from the Digital Access to a Sky Century @ Harvard (DASCH) [10, 11], AAVSO [19], and the Catalina Real-time Transient Survey [6] databases, supplemented by the data of $[14,8,22]$. We characterize the long-term light curve of $\mathrm{X}$ Ser and interpret it in the context of behaviors of other old novae.

Although DASCH contains only the upper limits of brightness of this pre-nova, it shows that this object was significantly fainter than the early post-nova. Especially a lower envelope of the data of the post-nova is considerably fainter after JD 2452000 than in the early post-nova near JD 2418500 (Figs.1 and 2a). We interpret it as significantly divergent mass transfer rates from the donor to the WD in these two epochs. X Ser displays strong complex activity with the characteristics of various $\mathrm{CV}$ types after the return to quiescence from its classical nova outburst (Fig.1).

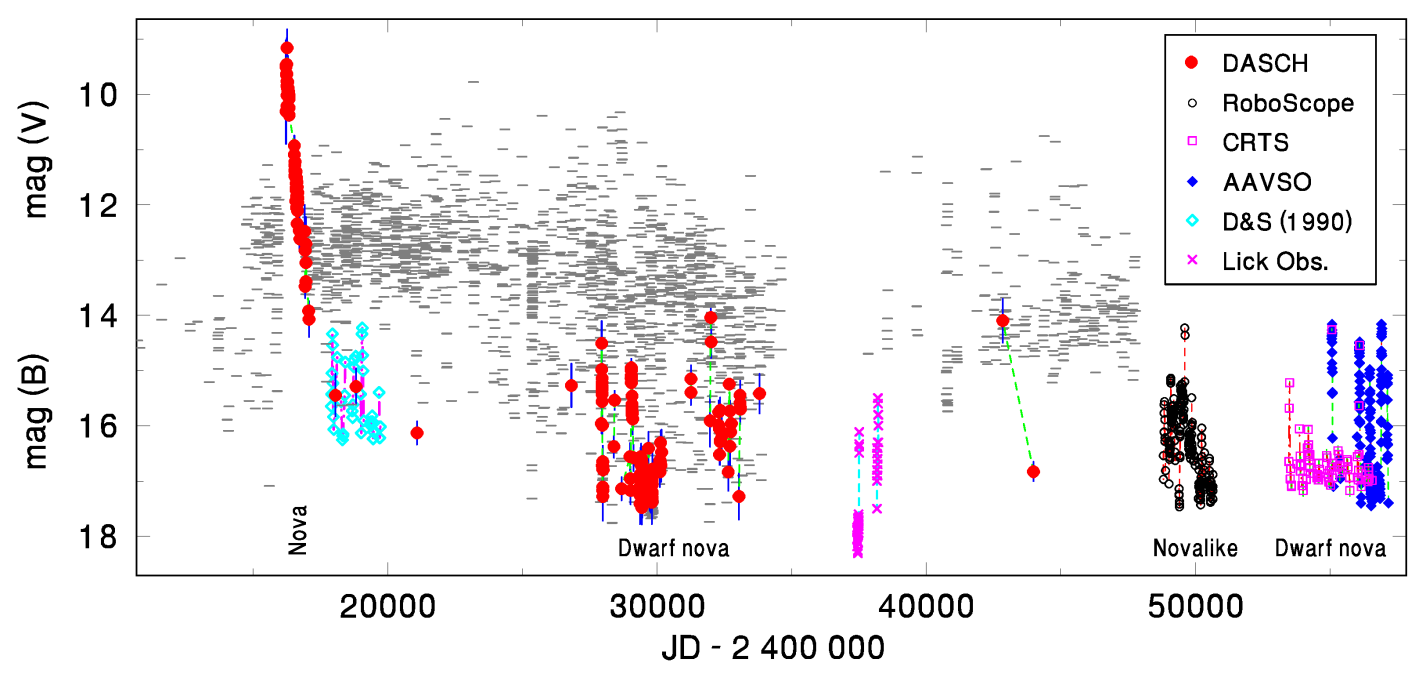

Figure 1: Long-term activity of X Ser. Data from several databases were merged: DASCH [10], RoboScope [14], the Catalina Survey [6], AAVSO [19], [8] and [22]. The short horizontal lines represent the upper limits of brightness if X Ser was not detected. The error bars of the data points are marked. The points were connected by a line in the densely covered parts of the light curves to guide the eye. See [39] for more.

Figure 2a shows a long classical nova outburst of X Ser. A linear fit to the decaying branch in which the fluctuations were not visible satisfies the profile of the light curve. The segment of the 

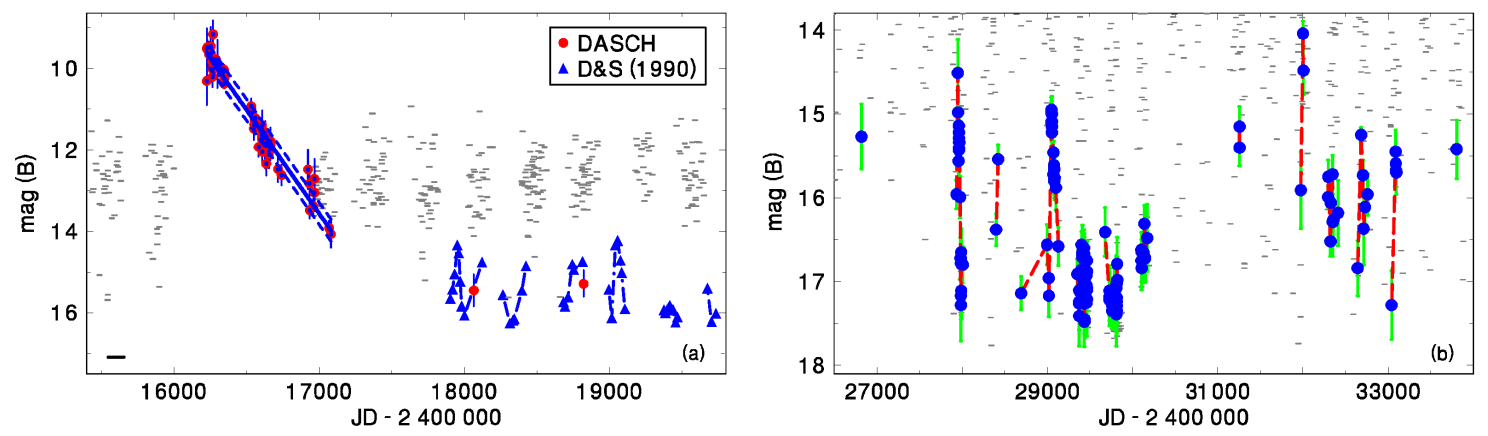

Figure 2: (a) The classical nova outburst in X Ser (data from DASCH [10] and [8]). A linear fit to the decaying branch in which the fluctuations were not visible is shown. The brightness of the fluctuations after JD 2417800 was significantly higher than the extension of this linear fit. (b) Segment with large variations of brightness between outbursts (flares) of the post-nova (DASCH data). The error bars of the data points are marked. The points were connected by a line in the densely covered parts of the light curves to guide the eye. The short horizontal lines represent the upper limits of brightness if X Ser was not detected on the plate.

data obtained after JD 2417800 shows the fluctuations whose brightness is significantly higher than an extension of this linear fit. Also a lower envelope of brightness of these fluctuations in Fig. 2a was significantly brighter than in the later phases (Fig. 1). We conclude that it is not possible to precisely determine the boundary between the end of the classical nova outburst and the start of the early post-nova.

$\mathrm{X}$ Ser displays complex time evolution of activity. In the interpretation, several mechanisms for the long-term activity appear to operate. A segment with large variations of brightness even between the outbursts (flares) of the post-nova in Fig. $2 \mathrm{~b}$ shows that these events were superimposed on the large, more gradual variations of brightness occurring on the timescale of hundreds of days. We interpret it as a slow decrease and fluctuations of the mass transfer rate from the donor with time.

A series of isolated brightenings from a well defined level of brightness in the later post-nova is displayed in Fig. 3. The decaying branches of these individual post-nova outbursts display large mutual similarities. They constitute a uniform group. We interpret these events as the dwarf nova (DN) outbursts. These decaying branches can be explained by propagation of cooling front (models of $[36,12])$ through the accretion disk.

We interpret the slow rising branches of the events in Fig. 3 as the inside-out outbursts (heating front propagating from the inner disk region outward (see [36] for model). The largely different peak magnitudes of the individual events suggest that heating front may not reach the outer disk rim in some outbursts.

The well defined decaying branch of an event in [39] speaks in favor of some episodic brightenings in DASCH data consistent with the DN outbursts. It is thus likely that the DN outbursts started from the quiescent level with the largely variable brightness. This quiescent level became more stable only after JD 2453000.

A big bump near JD 2450000 in Fig. 1 is different from the isolated brightenings which we interpret as the DN outbursts. This bump is much longer than any of these DN outbursts. Also its decaying branch is much less steep than those of the DN outbursts in Fig. 3. We ascribe this bump 


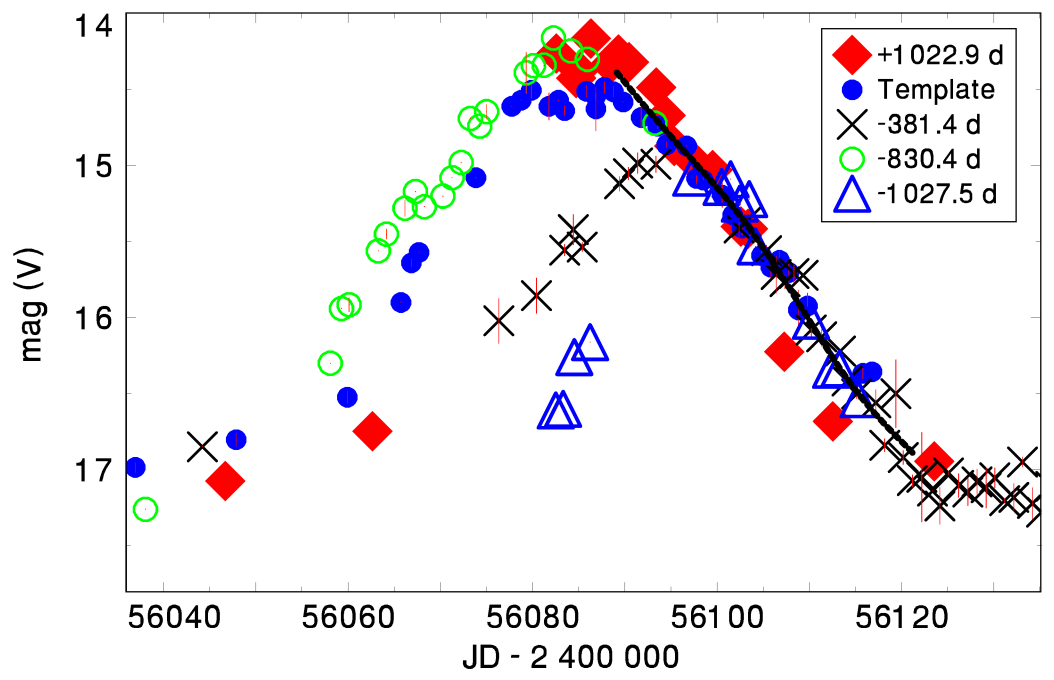

Figure 3: Comparison of outbursts in the post-nova X Ser (the Catalina Survey [6] and CCD AAVSO [19] data). They were aligned according to their decaying branches to match the decaying branch of the template. The time of crossing the brightness $15.5 \mathrm{mag}(V)$ and the time shifts with respect to the template are listed. The dashed line is a HEC13 fit to the ensemble of decaying branches of these outbursts. HEC13 was written by [13] and is based on the method of [45].

to a burst of mass outflow from the donor influenced by the outburst of the classical nova. The slow decaying branch of this bump can be explained as a decrease of the mass inflow into an ionized accretion disk.

In the interpretation, the post-nova X Ser rapidly transitioned to the thermal-viscous instability (TVI) regime of the accretion disk, initially only intermittently. The occurrence of the DN outbursts shortly after the end of the classical nova outburst suggests that the mass transfer rate into the disk was usually not sufficiently high to prevent the TVI of this post-nova. The very long $P_{\mathrm{orb}}$, and hence the large accretion disk of X Ser can contribute to this. More details about the activity of X Ser can be found in [39].

\section{V446 Her}

V446 Her exploded as a classical nova in 1960 [4]. It is a CV with $P_{\text {orb }}$ of $4.97 \mathrm{hr}$ [43]. This post-nova displayed a series of DN outbursts [15].

Figure 4 shows the characteristics of various CV types which V446 Her displayed during time. While the large-amplitude fluctuations of brightness of the pre-nova (see also a detail in [27], indicating a profile of a DN outburst) suggest the TVI of the accretion disk, the bright state of the early post-nova [40] was only slightly fainter than the peak of a DN outburst in the pre-nova. It is consistent with a standstill in the ZCam DNe or a high state in novalikes. This suggest that the accretion disk was in the ionized state [46]. The TVI of the disk then re-appeared several years after the end of the classical nova outburst (red open circles in Fig. 4 (data of [15])).

The outbursts of V446 Her [15] display big mutual similarities in their decaying branches no matter how bright their peak magnitudes and durations are (Fig. 5). This can be explained as 


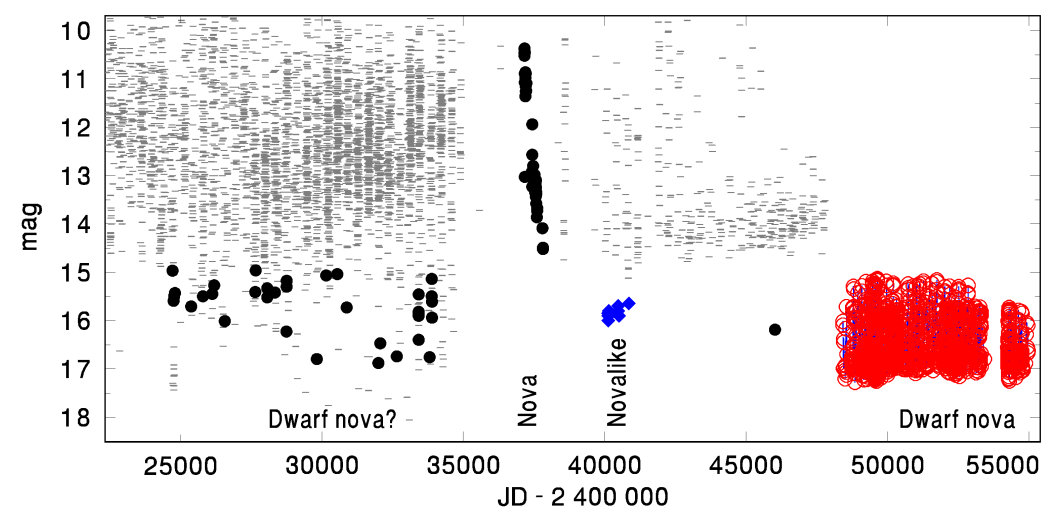

Figure 4: Long-term activity of V446 Her. Data from several databases were merged: DASCH [10] (black closed circles), photographic plates of [40] (blue closed diamonds), RoboScope CCD data [15] (red open circles). The short horizontal lines represent the upper limits of brightness. The error bars of the data points are marked. The points were connected by a line in the densely covered parts of the light curves to guide the eye.

propagation of cooling front across the disk [36]. The viscous plateau in which the whole disk becomes fully ionized occurs only during the peaks of some outbursts. The duration of these plateaux is highly variable.

The profiles of the rising branches in Fig. 5 are considerably variable, which suggests various types of the DN outbursts in V446 Her. We interpret the fast rising branches as the outside-in outbursts (heating front starting in the outer disk region and propagating inward (it always brings the whole disk to the hot (ionized) state (see model in [36])). In this regard, the slow rising branches of some events in Fig. 5 are the inside-out outbursts (heating front propagates from the inner disk region outward (it may not reach the outer disk rim) (model of [36]).

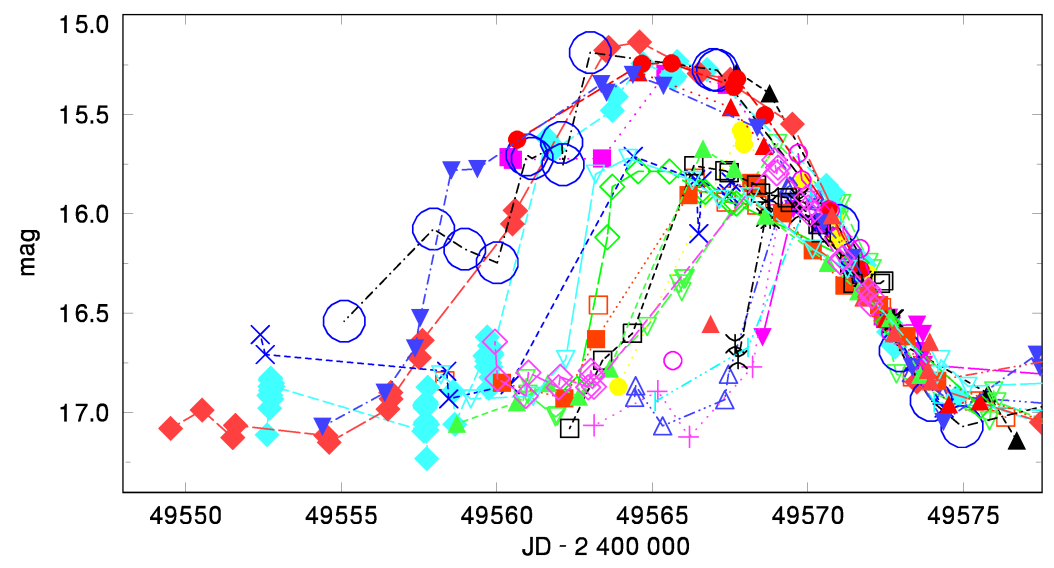

Figure 5: Comparison of outbursts in V446 Her (CCD data from [15]) during the post-nova stage. The individual outbursts were shifted along the time axis to match the decaying branch of the template. 


\section{V1363 Cyg}

V1363 Cyg, classified as a DN in the catalogue of [3], is also a post-nova because it is approximately centered on a nebula with about 2 arcmin diameter, ascribed to the ejecta of a nova outburst [29]. A typical duration of detectability of a nova shell is 100-200 years [29] but this depends on several factors, especially on the distance of the nova. There are also some CVs (ZCam, the lower limit of 1300 years [32]; AT Cnc, about 330 years ago [33]; Nova Sco 1437, 583 years ago [34]) which have observable shells older than 200 years. This constrains the upper limit of the time elapsed since the nova outburst. The nova explosion of V1363 Cyg was thus a relatively recent event.
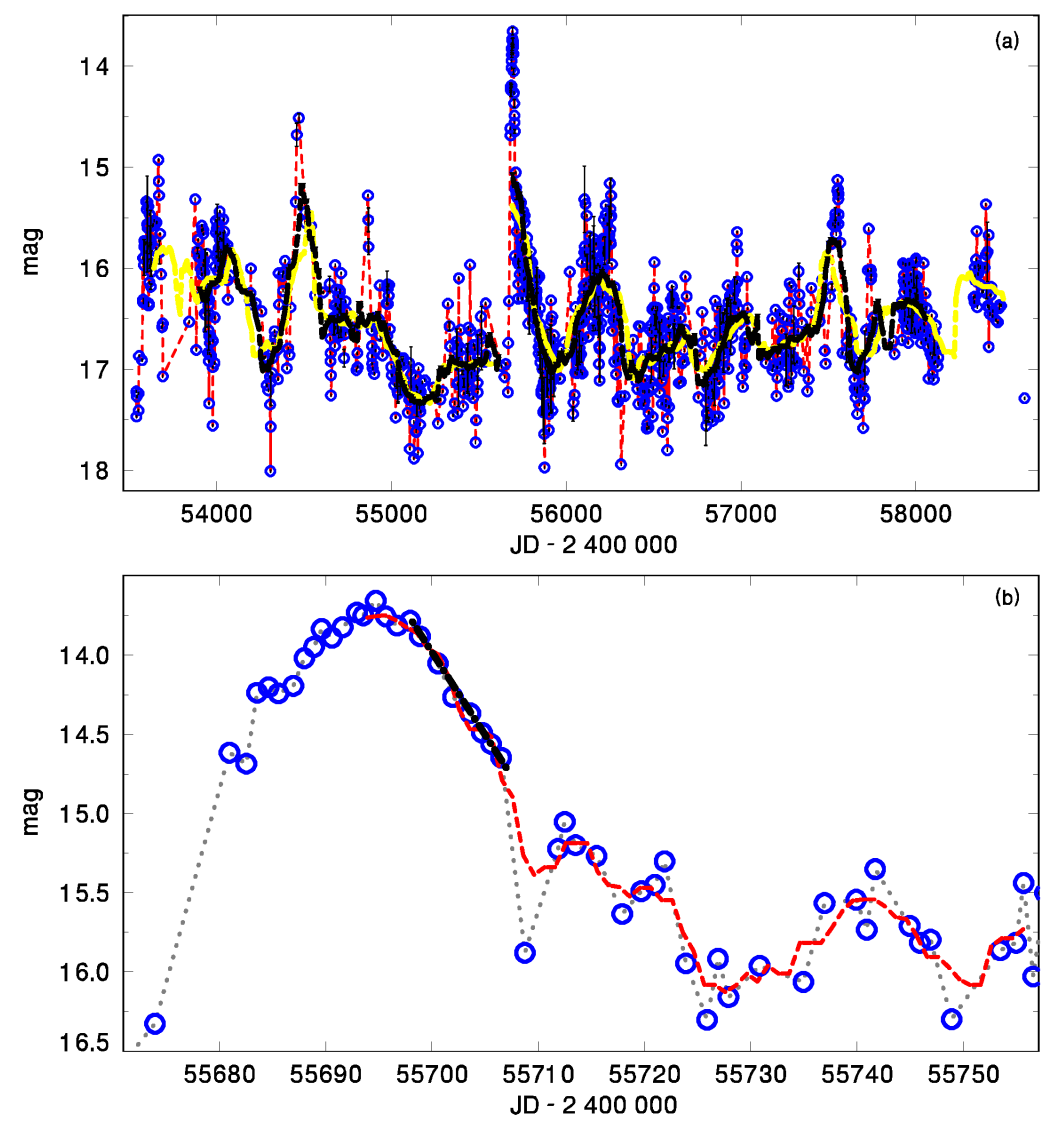

Figure 6: (a) Long-term activity of V1363 Cyg (AAVSO CCD V-band data [20]). The error bars are marked but they are often smaller than the size of the symbol. The points were connected by a line in the densely covered parts of the light curve to guide the eye. The moving averages are marked as the black line for the filter half-width $Q=100 \mathrm{~d}$ and as the yellow line for $Q=60 \mathrm{~d}$. (b) Detail of an outburst. The black straight line shows the decaying branch used for determining of the decay rate. The red dashed line marks the moving averages $(Q=3 \mathrm{~d})$ of the decaying branch and the subsequent fluctuations of brightness.

The long-term activity of V1363 Cyg consists of a series of large-amplitude fluctuations (Fig. 6a). The dominant brightness variations occur on the timescale of days and weeks. To emphasize the long-term fluctuations of brightness, the two-sided moving averages (method of [2]) of the data were made for the filter half-widths $Q=100 \mathrm{~d}$ and $Q=60 \mathrm{~d}$. A detail of the brightest peak (out- 
burst, flare) in Fig. 6b shows that the night-to-night fluctuations were suppressed and started only in the late phase of this event. In the interpretation, this event was a DN outburst (propagation of heating and cooling fronts in the accretion disk [36, 12]). Its relation to the properties of the outbursts in DNe is shown in Fig. 8.

The weighted wavelet Z-transform (WWZ) (method of [9]) of the moving averages of the brightness variations with $Q=60 \mathrm{~d}$ from Fig. 6a was made. It shows a time evolution of fluctuations of brightness (Fig. 7). The value of WWZ indicates whether or not there is a periodic fluctuation at a given time at a given frequency. Although these fluctuations are cyclic (Fig. 7b), this cycle is unstable and intermittently present.

In the interpretation, also activity of the mass-donating star can contribute to the brightness variations of V1363 Cyg beside the DN outburst(s). It can be caused by the appearance and changes of the position of the active regions (loops [18], starspots [24]) with respect to the L1 point by a differential rotation of the donor [31].

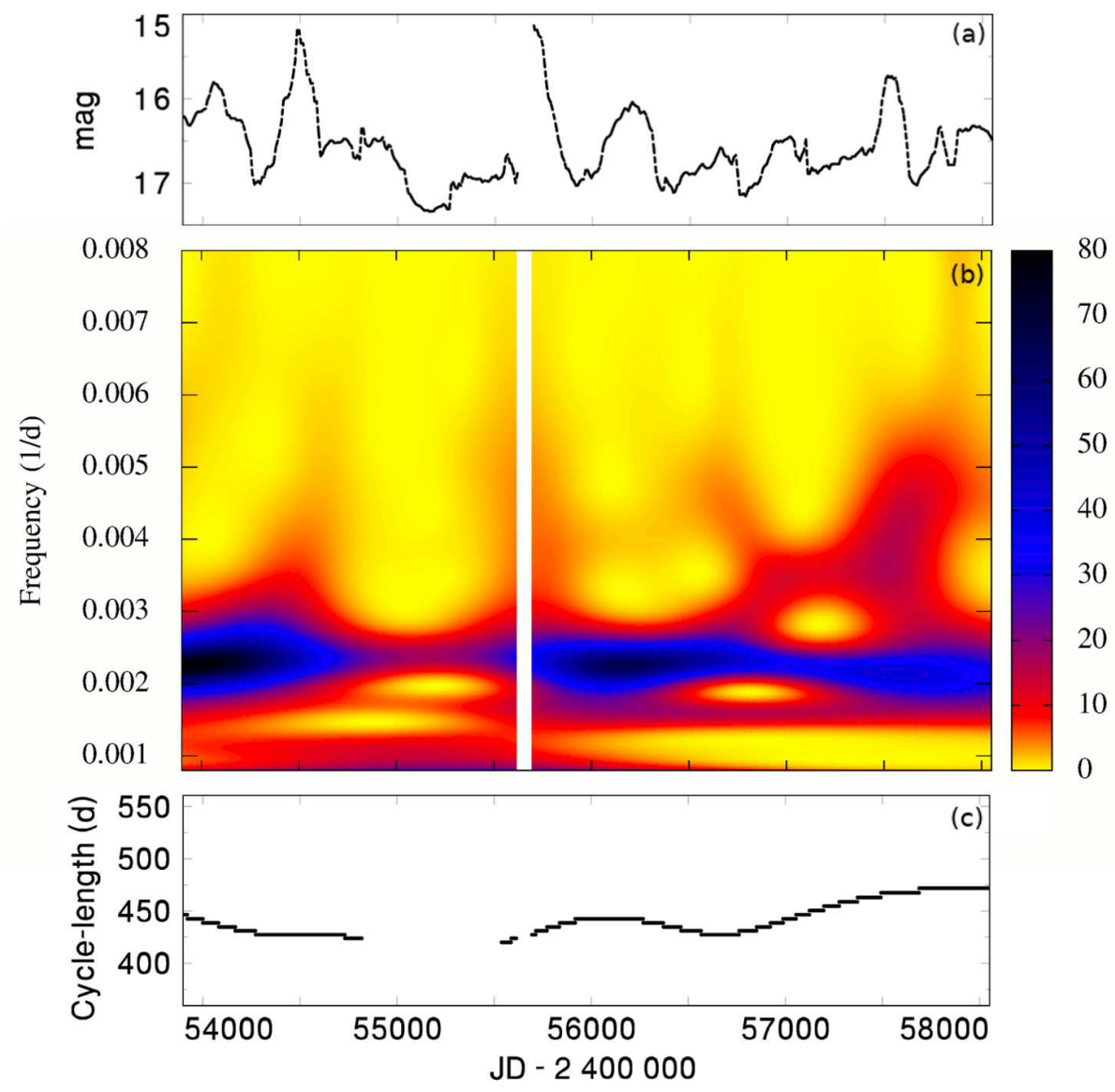

Figure 7: (a) The moving averages of the brightness variations of V1363 Cyg from Fig. 6a (AAVSO CCD data). (b) Cycles in these changes of brightness (the moving averages with $Q=60 \mathrm{~d}$ ) from panel (a). The WWZ-transform (method of [9]) was used. Frequency is given in $\mathrm{d}^{-1}$. The color scale represents the values of WWZ. The higher the WWZ value, the better defined the cycle-length. (c) The best cycle-length measured in days. 


\section{GK Per}

GK Per (Nova Per 1901) [7] is an intermediate polar [47] with $P_{\text {orb }}=1.99 \mathrm{~d}$ [5]. This length is very long among CVs. Fluctuations of brightness by about $1 \mathrm{mag}$ on the time scale of months appeared after the return to quiescence. The outburst of this classical nova gave rise to a novalike post-nova. Later, the discrete optical outbursts appeared (e.g. [28, 17, 38]). Model of the TVI of the accretion disk was able to reproduce the basic features of the outbursts [21]. A DN outburst of GK Per is included in Fig. 8.

The DN outbursts of GK Per appeared only when the TVI could operate because the cooled WD was already not able to irradiate the accretion disk sufficiently to keep it in the hot (ionized) state. This enabled the accretion disk to switch between the hot and cool states, as modeled by [30].

\subsection{The decay rate of the DN outbursts in post-novae}

The light curves of outbursts (flares) of post-novae are helpful in determining the mechanisms which cause them. The decay rate is an important parameter of the light curves of the DN outbursts. The quantity $\tau_{\mathrm{D}}$ is the decay rate expressed in days for a decrease of brightness by 1 mag [1].

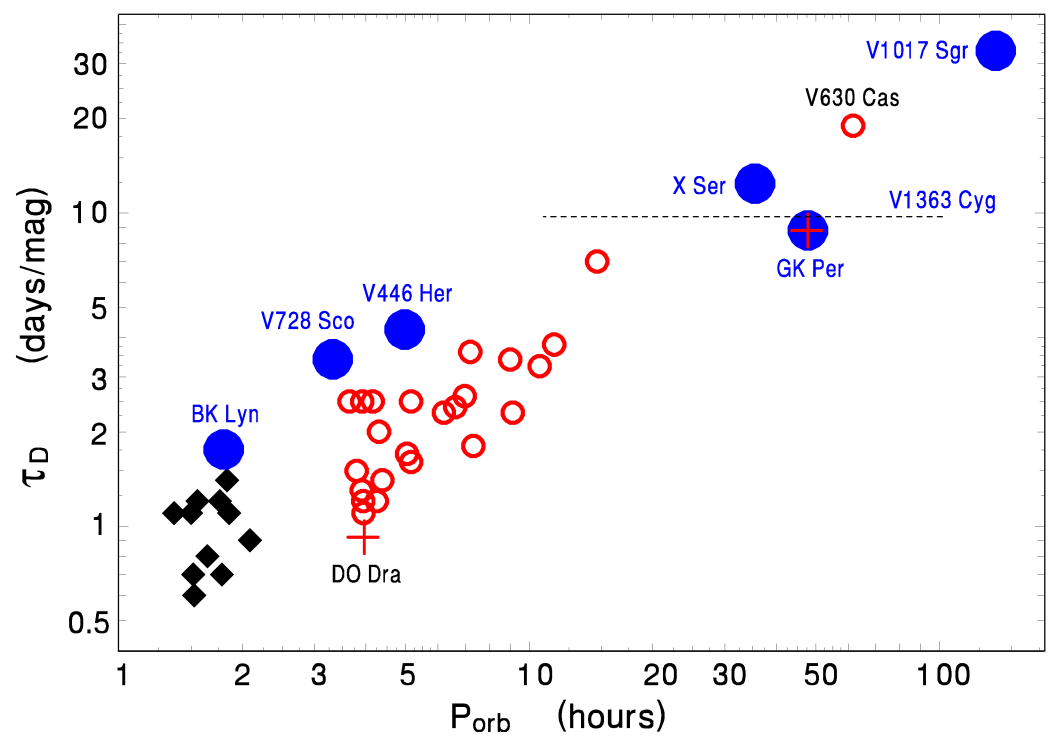

Figure 8: Decay rate of DN outbursts, $\tau_{\mathrm{D}}$, vs $P_{\mathrm{orb}}$. The outbursts in post-novae are marked by the solid circles. Crosses mark the intermediate polars. Diamonds represent the SU UMa systems. The dashed horizontal line marks the potential positions of V1363 Cyg, whose $P_{\text {orb }}$ is unknown. The data come from [46], [37], [15], [35] and [39].

Positions of various DNe are shown in Fig. 8 where $\tau_{\mathrm{D}}$ is plotted versus $P_{\text {orb }}$. This plot enables to assess how much a given outburst obeys the Bailey relation [1]. Most data come from [46] and [35]. Please note that the values of $\tau_{\mathrm{D}}$ of DO Dra [37] and GK Per [38, 39] are smaller than of most DNe with comparable $P_{\text {orb }}$. These two CVs are known to be the intermediate polars according to [25, 47]. The value of $\tau_{\mathrm{D}}$ of BK Lyn (probable Nova Lyn 101 [16]) was determined from the light curve of an outburst in this post-nova displayed in [26]. The value of $\tau_{\mathrm{D}}$ of V725 Sco (Nova Sco 1862 [42], $P_{\text {orb }}=3.32 \mathrm{hr}$ [41]) was determined from the light curve of an outburst plotted in [44]. 
The value of $\tau_{\mathrm{D}}$ of the outburst (flare) in V1363 Cyg in Fig. 6b suggests that it can be a DN outburst in a CV with a very long $P_{\text {orb }}$, about $10-100 \mathrm{~h}$, taking into account a significant uncertainty in fitting the light curve with a scatter.

Figure 8 shows that the outbursts of post-novae are consistent with those of "normal" DNe (i.e., the systems whose classical nova outbursts were not observed). This speaks in favor of the DN outburst nature of the episodic brightenings of these post-novae. This also suggests that the accretion disks of these post-novae are not ionized all the time. They were able to switch from the hot (ionized) state to the TVI regime.

The positions of post-novae with $P_{\text {orb }}<6 \mathrm{hr}$ in Fig. 8 suggest that their $\tau_{\mathrm{D}}$ is slightly bigger in comparison with DNe of the same $P_{\text {orb }}$ but without a classical nova outburst observed in the past. This can be caused by a very hot WD as a result of the relatively recent classical nova outburst. A hot source of emission can be indicated by the small amplitude of the DN outbursts in post-novae (1.5-1.8 mag in V446 Her (Fig. 5), a similar situation in BK Lyn [26]). This TVI in post-novae with very hot WD and possibly also with hot inner disk regions is worth modeling.

\section{Conclusions}

A big range of optical luminosities of post-novae exists. The discrete features (e.g. outbursts, flares) of their long-term activity are common. Also large changes of the type of activity in the decades after the classical nova outburst are possible.

The similarities in the decaying branches of the outbursts in post-novae and the relation of their $\tau_{\mathrm{D}}$ to $P_{\text {orb }}$ favor the DN outbursts. They are caused by propagation of cooling front across the accretion disk.

Features consistent with the DN outbursts appear in the later phases of the gradual decay of the mean luminosity of some post-novae. This suggests that some post-novae contain the conditions for the onset and existence of the accretion disk with the TVI. Such a TVI of the disk can appear in some post-novae even during the first century of the classical nova outburst (often sooner after the classical nova outburst in $\mathrm{CVs}$ with longer $P_{\text {orb }}$ ). Post-novae with larger accretion disks can thus possibly get to the TVI zone more easily.

Also activity of the mass-donating star can contribute to the brightness variations of some post-novae. It can be caused by the appearance and changes of the position of the active regions (loops [18], starspots [24]) with respect to the L1 point by a differential rotation of the donor [31].

Acknowledgments This study was supported by these projects: EU project H2020 871158, grant No. 17-05840S provided by the Grant Agency of the Czech Republic, the project RVO:67985815. This research has made use of the observations from the DASCH project at Harvard, partially supported from NSF grants AST-0407380, AST-0909073, and AST-1313370. This work also used the data from the Catalina Transient Survey, AAVSO International database (USA), and the AFOEV database (France). I thank the variable star observers worldwide. The public data from Swift/BAT transient monitor provided by the Swift/BAT team were used. I used the code developed by Dr. G. Foster and available at ww.aavso.org/winwwz . I also thank Prof. Petr Harmanec for providing me with the code HEC13. The Fortran source version, compiled version and brief instructions on how to use the program can be obtained at http://astro.troja.mff.cuni.cz/ftp/hec/HEC13/ 


\section{References}

[1] J. Bailey, Journ. Brit. Astr. Ass., 86, 30 (1975).

[2] P. J. Brockwell, \& R. A. Davis, Time Series: Theory and Methods, Springer-Verlag New York Inc. (1987).

[3] A. Bruch, F.-J. Fischer, U. Wilmsen, $A \& A S$, 70, 481 (1987).

[4] Thomas A. Cragg, PASP, 72, 475 (1960).

[5] D. Crampton, A. P. Cowley, W. A. Fisher, ApJ, 300, 788 (1986).

[6] A. J. Drake, et al., ApJ, 696, 870 (2009).

[7] H. W. Duerbeck, $\operatorname{SSRv}, 45,1$ (1987).

[8] H. W. Duerbeck \& W. C. Seitter, Physics of Classical Novae. Proceedings of Colloquium No.122 of the International Astronomical Union, held in Madrid, Spain, on June 27-30, 1989. Editors, A. Cassatella, R. Viotti; Publisher, Springer-Verlag, Berlin, Germany; New York, NY. Part of the Lecture Notes in Physics book series (1990) (LNP, Vol. 369, p. 165)

[9] G. Foster, AJ, 112, 1709 (1996).

[10] J. Grindlay, S. Tang, E. Los, M. Servillat, IAUS, 285, 29 (2012a).

[11] J. E. Grindlay \& R. E. Griffin, IAUS, 285, 243 (2012b).

[12] J.-M. Hameury, K. Menou, G. Dubus, J.-P. Lasota, J.-M. Hure, MNRAS, 298, 1048 (1998).

[13] P. Harmanec, (1992). http://astro.troja.mff.cuni.cz/ftp/hec/HEC13/

[14] R. K. Honeycutt, J. W. Robertson, G. W. Turner, AJ, 115, 2527 (1998).

[15] R. K. Honeycutt, J. W. Robertson, S. Kafka, AJ, 141, 121 (2011).

[16] T.-T. Hsi, Smithson. Contr. Astrophys., 2, 109 (1958).

[17] R. Hudec, Bull. Astron. Inst. Czechosl., 32, 93 (1981).

[18] S. Kafka, T. Ribeiro, R. Baptista, R. K. Honeycutt, J. W. Robertson, ApJ, 688, 1302 (2008).

[19] S. Kafka, Obs. from the AAVSO Int. Database, www.aavso.org (2016).

[20] S. Kafka, Obs. from the AAVSO Int. Database, www.aavso.org (2019).

[21] Soon-Wook Kim, J. C. Wheeler, S. Mineshige, ApJ, 384, 269 (1992).

[22] T. D. Kinman, C. A. Wirtanen, K. A. Janes, ApJS, 11, 223 (1965).

[23] H. Leavitt, Harv. Circ. 142 (1908).

[24] M. Livio, \& J. E. Pringle, ApJ, 427, 956 (1994).

[25] J. Patterson, D. A. Schwartz, J. P. Pye, W. P. Blair, G. A. Williams, J.-P. Caillault, ApJ, 392, 233 (1992).

[26] Joseph Patterson, et al., MNRAS, 434, 1902 (2013).

[27] E. L. Robinson, AJ, 80, 515 (1975).

[28] F. Sabbadin, \& A. Bianchini, $A \& A S$, 54, 393 (1983).

[29] D. I. Sahman, V. S. Dhillon, C. Knigge, T. R. Marsh, MNRAS, 451, 2863 (2015). 
[30] M. R. Schreiber, B. T. GÃd'nsicke, J. K. Cannizzo, A\&A 362, 268 (2000).

[31] E. T. Scharlemann, ApJ, 253, 298 (1982).

[32] Michael M. Shara, Trisha Mizusawa, David Zurek, Christopher D. Martin, James D. Neill, Mark Seibert, ApJ, 756, 107 (2012).

[33] Michael M. Shara, Laurent Drissen, Thomas Martin, Alexandre Alarie, F. Richard Stephenson, MNRAS, 465, 739 (2017).

[34] M. M. Shara, et al., Natur, 548, 558 (2017).

[35] J. Shears, \& G. Poyner, JBAA, 120, 169 (2010).

[36] J. Smak, AcA, 34, 161 (1984).

[37] V. Šimon, $A \& A, \mathbf{3 6 0}, 627$ (2000).

[38] V. Šimon, $A \& A, \mathbf{3 8 2}, 910$ (2002).

[39] V. Šimon, $A \& A, \mathbf{6 1 4}$, id.A141, 7 pp. (2018).

[40] F. M. Stienon, PASP, 83, 363 (1971).

[41] C. Tappert, N. Vogt, L. Schmidtobreick, A. Ederoclite, J. Vanderbeke, MNRAS, 431, 92 (2013).

[42] J. Tebbutt, MNRAS, 38, 330 (1878).

[43] J. R. Thorstensen, \& C. J. Taylor, MNRAS, 312, 629 (2000).

[44] N. Vogt, C. Tappert, E. C. Puebla, I. Fuentes-Morales, A. Ederoclite, L. Schmidtobreick, MNRAS, 478, 5427 (2018).

[45] J. Vondrák, Bull. Astron. Inst. Czechosl., 20, 349 (1969).

[46] B. Warner, Cataclysmic Variable Stars, Cambridge Univ. Press, Cambridge (1995).

[47] M. G. Watson, A. R. King, J. Osborne, MNRAS, 212, 917 (1985). 